\title{
Delayed Stroke Treatment during COVID-19 Pandemic in China
}

\author{
Shiyuan Gua,b Zhengze Dai ${ }^{c}$ Huachao Shen ${ }^{d}$ Yongjie Baie ${ }^{\text {Xiaohao Zhang }}{ }^{f}$ \\ Xinfeng Liu ${ }^{a, f}$ Gelin Xua ${ }^{a, f}$ \\ aDepartment of Neurology, Jinling Clinical College of Nanjing Medical University, Nanjing, China; ${ }^{b}$ Department of \\ Neurology, Affiliated Yixing Hospital of Jiangsu University, Yixing, China; 'Department of Neurology, The Fourth \\ Affiliated Hospital of Nanjing Medical University, Nanjing Pukou Hospital, Nanjing, China; ${ }^{d}$ Department of \\ Neurology, BenQ Medical Center, Affiliated BenQ Hospital of Nanjing Medical University, Nanjing, China; \\ eDepartment of Neurology, First Affiliated Hospital and College of Clinical Medicine of Henan University of Science \\ and Technology, Luoyang, China; 'Department of Neurology, Jinling Hospital, Medical School of Nanjing University, \\ Nanjing, China
}

\section{Keywords}

Acute stroke · COVID-19 - Intravenous thrombolysis .

Treatment delay

\section{Abstract}

Background: Social distance, quarantine, pathogen testing, and other preventive strategies implemented during $\mathrm{CO}$ VID-19 pandemic may negatively influence the management of acute ischemic stroke (AIS). Objective: The current study aimed to evaluate the impacts of COVID-19 pandemic on treatment delay of AIS in China. Methods: This study included patients with AIS admitted in 2 hospitals in Jiangsu, China. Patients admitted before and after the COVID-19 pandemic outbreak (January 31, 2020, as officially announced by the Chinese government) were screened to collect sociodemographic data, medical history information, and symptom onset status from clinical medical records and compared for pre- (measured as onset-to-door time [ODT]) and posthospital delay (measured as door-to-needle time [DNT]). The influencing factors for delayed treatment (indicated as onset-toneedle time $>4.5 \mathrm{~h}$ ) were analyzed with multivariate logistic regression analysis. Results: A total of 252 patients were in- cluded, of which 153 (60.7\%) were enrolled before and 99 (39.3\%) after the COVID-19 pandemic. ODT increased from $202 \mathrm{~min}$ (interquartile range [IQR] 65-492) before to $317 \mathrm{~min}$ (IQR 75-790) after the COVID-19 pandemic $(p=0.001)$. DNT increased from $50 \mathrm{~min}$ (IQR 40-75) before to $65 \mathrm{~min}$ (IQR 4884 ) after the COVID-19 pandemic $(p=0.048)$. The proportion of patients with intravenous thrombolysis in those with AIS was decreased significantly after the pandemic $(15.4 \%$ vs. $20.1 \% ; p=0.030$ ). Multivariate logistic regression analysis indicated that patients after COVID-19 pandemic, lower educational level, rural residency, mild symptoms, small artery occlusion, and transported by other means than ambulance were associated with delayed treatment. Conclusions: $\mathrm{CO}$ VID-19 pandemic has remarkable impacts on the management of AIS. Both pre- and posthospital delays were prolonged significantly, and proportion of patients arrived within the 4.5-h time window for intravenous thrombolysis treatment was decreased. Given that anti-COVID-19 measures are becoming medical routines, efforts are warranted to shorten the delay so that the outcomes of stroke could be improved. (c) 2021 S. Karger AG, Basel karger@karger.com www.karger.com/ced

(c) 2021 S. Karger AG, Basel

Karger"
Correspondence to:

Xinfeng Liu, xfliu2@vip.163.com

Gelin Xu, gelinxu@nju.edu.cn 


\section{Introduction}

Recently developed treatments, such as intravenous thrombolysis and mechanical thrombectomy, can significantly improve the outcomes of acute ischemic stroke (AIS). But, the effects of these treatments were highly time-dependent, which emphasize the importance of rapid pre- and posthospital managements. For selected patients with onset-to-needle time (ONT) shorter than 4.5 $\mathrm{h}$, intravenous thrombolysis could be applied. But, those with ONT shorter than $3 \mathrm{~h}$ had a higher likelihood of a 90-day favorable outcome [1]. For selected patients with onset-to-puncture time (OPT) shorter than $6 \mathrm{~h}$, mechanical thrombectomy could be applied. Although patients with OPT between 6 and $24 \mathrm{~h}$ still could be screened for mechanical thrombectomy, the effects attenuate rapidly with time delay. Current guidelines recommended that extra imaging examinations should be performed for selecting patients with OPT between 6 and $24 \mathrm{~h}$ for mechanical thrombectomy $[2,3]$. Therefore, when applying intravenous thrombolysis and mechanical thrombectomy in AIS patients, the shorter the treatment delays, the better the functional outcomes.

Since the outbreak of COVID-19 pandemic, China has implemented several nation-wide strategies for preventing and containing the spread of the disease [4]. Social distance, quarantine, pathogen testing, and other strategies were taken from January 31,2020 , as officially announced by the Chinese government. These measures influenced not only the regular medical procedures but also the health-seeking behaviors. All these changes may have influenced the management of stroke, but the impacts are largely undetermined [5]. This study aimed to explore the impact and extent of COVID-19 pandemic on treatment delay of AIS in China. Additionally, we probed potential factors responsible for the treatment delay.

\section{Methods}

\section{Study Design and Patient Population}

This study is a part of an ongoing program for analyzing preand posthospital delay in managing stroke patients. Patients with acute stroke were enrolled in 2 tertiary hospitals in Jiangsu province. On January 31, 2020, the Chinese government announced several nation-wide strategies for preventing the COVID-19 pandemic. Patients with AIS diagnosed during December 1, 2019, and January 30, 2020 (pre-COVID-19), and those diagnosed during February 1, 2020, and March 31, 2020 (post-COVID-19), were compared in this study. AIS was diagnosed based on clinical symptoms and computed tomography or magnetic resonance imaging scans. Patients who reached the hospitals within 7 days after stroke onset were included. Socioeconomic status, medical history, and stroke symptoms were reviewed and analyzed. All participants and their relatives provided written informed consent, and the study was approved by the Ethics Committees of the participated hospital. The work was supported by the National Natural Science Foundation of China (NSFC 81870947).

\section{Treatment Delay Assessment}

Demographic and clinical data were collected after hospitalization. Onset-to-door time (ODT) was defined as the duration from stroke symptom onset or time last known well to hospital arrival, which included awareness time, decision time, and transporting time. Decision time is defined as the duration from symptom onset to the decision being made to go to hospital. Door-to-needle time (DNT) was defined as the time from hospital arrival to the start of intravenous thrombolysis from hospital arrival to the start of intravenous thrombolysis. For those who did not meet the criteria of intravenous thrombolysis, DNT was based on a presumed thrombolytic therapy of the earliest possibility. Door-to-puncture time was defined as the duration from hospital arrival to groin puncture for mechanical thrombectomy. For those who did not meet the criteria of mechanical thrombectomy, door-to-puncture time was based on a presumed mechanical thrombectomy of the earliest possibility. Potential influencing factors for treatment delay, such as residency, means of transportation, and the level of the first visited hospital, were retrieved and analyzed. Self-management after stroke onset means that patients choose to stay at home or take medicine on their own, instead of going to the hospital immediately. Etiology was classified using the Trial of ORG 10172 in Acute Stroke Treatment categories. The severity of stroke was measured with the National Institutes of Health Stroke Scale (NIHSS). In this study, we choose $4.5 \mathrm{~h}$ as the cut point for defining delayed treatment because $4.5 \mathrm{~h}$ is the accepted deadline for rt-PA intravenous thrombolysis at present [1].

\section{Statistical Analysis}

Continuous variables were expressed as mean \pm standard deviation or median and interquartile range (IQR) as appropriate. Categorical variables were presented as frequency and percentage. Continuous variables with normal distribution were compared using Student's $t$-test. The $\chi^{2}$ and Fisher's exact tests were used for comparing categorical values. Multiple-variable stepwise logistic regression was used to determine the main influencing factors of treatment delay. A 2 -sided $p$ value of $<0.05$ was deemed as statistical significance. All statistical analyses were performed using SPSS 25.

\section{Results}

A total of 252 patients were included during the described time frames, of which 153 (60.7\%) were arrived before and 99 (39.3\%) after the COVID-19 pandemic. The mean age of the included patients was $69.1 \pm 11.3$ years, and $167(62.5 \%)$ of them were male. The median (IQR) NIHSS score at admission was $6(3-13)$ in the preCOVID-19 group and 8 (5-16) in the post-COVID-19 
Table 1. Characteristics and treatment delay of stroke patients before and after COVID-19 pandemic

\begin{tabular}{|c|c|c|c|}
\hline Characteristics & Pre-COVID-19, $n=153$ & Post-COVID-19, $n=99$ & $p$ value \\
\hline Age, year, mean & $69.5 \pm 11.1$ & $70.1 \pm 12.2$ & 0.334 \\
\hline Male gender, $n(\%)$ & $92(60.1)$ & $70(66.0)$ & 0.133 \\
\hline \multicolumn{4}{|l|}{ Education, $n(\%)$} \\
\hline Elementary education & $46(30.1)$ & $32(32.3)$ & \multirow{3}{*}{0.242} \\
\hline Secondary education & $86(56.2)$ & $54(54.4)$ & \\
\hline Higher education & $21(13.7)$ & $13(14.3)$ & \\
\hline Solitary, $n(\%)$ & $47(30.7)$ & $32(32.3)$ & 0.410 \\
\hline \multicolumn{4}{|l|}{ Residence, $n(\%)$} \\
\hline Urban & $103(67.3)$ & $70(70.7)$ & \multirow{2}{*}{0.411} \\
\hline Rural & $50(32.7)$ & $29(29.3)$ & \\
\hline Daytime onset, $n(\%)$ & $79(51.6)$ & $53(53.5)$ & 0.433 \\
\hline \multicolumn{4}{|l|}{ Stroke etiology, $n(\%)$} \\
\hline Large artery atherosclerosis & $59(38.5)$ & $38(38.3)$ & \multirow{5}{*}{0.270} \\
\hline Small vessel disease & $56(36.6)$ & $40(40.4)$ & \\
\hline Cardioembolism & $32(21.1)$ & $17(17.2)$ & \\
\hline Other demonstrated cause & $3(1.9)$ & $1(1.0)$ & \\
\hline Undetermined cause & $3(1.9)$ & $3(2.8)$ & \\
\hline NIHSS, median (IQR) & $6(3-13)$ & $8(5-16)$ & 0.040 \\
\hline Stroke history, $n(\%)$ & $33(21.6)$ & $22(22.2)$ & 0.871 \\
\hline Hypertension, $n(\%)$ & $100(65.4)$ & $60(60.6)$ & 0.640 \\
\hline Diabetes, $n(\%)$ & $60(39.2)$ & $42(42.4)$ & 0.256 \\
\hline Hyperlipidemia, $n(\%)$ & $50(32.6)$ & $31(31.3)$ & 0.734 \\
\hline Atrial fibrillation, $n(\%)$ & $20(13.1)$ & $15(15.1)$ & 0.399 \\
\hline Coronary heart disease, $n(\%)$ & $22(13.6)$ & $17(17.1)$ & 0.277 \\
\hline Smoking, $n(\%)$ & $68(44.4)$ & $40(40.5)$ & 0.143 \\
\hline Alcohol drinking, $n(\%)$ & $55(34.1)$ & $40(37.7)$ & 0.300 \\
\hline Self-management, $n(\%)$ & $63(41.1)$ & $63(63.6)$ & $<0.001$ \\
\hline Transported by ambulance, $n(\%)$ & $41(30.7)$ & $33(33.3)$ & 0.105 \\
\hline ODT, min, median (IQR) & $202(25-492)$ & $317(65-790)$ & 0.010 \\
\hline Decision time & $129(55-430)$ & $244(80-710)$ & $<0.001$ \\
\hline Transportation & $73(31-93)$ & $67(33-88)$ & 0.316 \\
\hline DNT, median (IQR), min, $n(\%)$ & $50(40-75)$ & $65(48-84)$ & 0.048 \\
\hline ONT $<4.5 \mathrm{~h}, n(\%)$ & $53(35.3)$ & $29(29.3)$ & 0.032 \\
\hline Intravenous thrombolysis, $n(\%)$ & $34(22.2)$ & $15(15.1)$ & 0.030 \\
\hline Mechanical thrombectomy, $n(\%)$ & $24(15.7)$ & $12(12.1)$ & 0.115 \\
\hline In-hospital mortality, $n(\%)$ & $8(4.9)$ & $6(6.1)$ & 0.120 \\
\hline Discharge mRS score $(0-2), n(\%)$ & $92(60.1)$ & $54(54.5)$ & 0.180 \\
\hline
\end{tabular}

IQR, interquartile range; NIHSS, National Institutes of Health Stroke Scale; ODT, onset-to door time; DNT, door-to-needle time; mRS, modified Ranking Scale; ONT, onset-to needle time.

group $(p=0.040)$. However, no significant differences concerning age, sex, residence, education level, onset time, medical insurance, etiology of ischemic stroke, ambulance use, and comorbidities were detected between patients who arrived before and after the COVID-19 pandemic (Table 1).

The ODT, a proxy of prehospital delay, was significantly longer in post- than pre-COVID-19 pandemic patients (317 [IQR 65-790] vs. 202 [IQR 25-492] min; $p=$ $0.010)$. The decision time for patients after the COVID-19 pandemic was significantly longer than that of those before COVID-19 pandemic (129 [IQR 55-430] vs. 244 [IQR 80-710] min, $p<0.001)$. Time used for transportation was similar between patients before and after the COVID-19 pandemic (67 [IQR 33-88] vs. 73 [IQR 31$93] \mathrm{min} ; p=0.316)$. DNT was prolonged significantly after the implementation of anti-pandemic strategies (65 [IQR 48-84] vs. 50 [IQR 40-75] $\mathrm{min}, p=0.048$ ). The proportion of patients choosing self-management after stroke onset was significantly increased after the pandem- 
Table 2. Influencing factors for delayed treatment

\begin{tabular}{|c|c|c|c|}
\hline \multirow[t]{2}{*}{ Characteristics } & \multicolumn{2}{|l|}{ ONT } & \multirow[t]{2}{*}{$p$ value } \\
\hline & $\leq 4.5 \mathrm{~h}, n=80$ & $>4.5 \mathrm{~h}, n=172$ & \\
\hline Age, yr, mean & $67.5 \pm 12.2$ & $70.1 \pm 11.8$ & 0.134 \\
\hline Male gender, $n(\%)$ & $51(63.8)$ & $106(61.6)$ & 0.736 \\
\hline \multicolumn{4}{|l|}{ Education, $n(\%)$} \\
\hline Elementary education & $15(18.4)$ & $64(37.2)$ & \multirow{3}{*}{0.018} \\
\hline Secondary education & $46(57.5)$ & $94(54.7)$ & \\
\hline Higher education & $19(24.1)$ & $14(8.1)$ & \\
\hline Solitary, $n(\%)$ & $21(26.3)$ & $58(33.7)$ & 0.044 \\
\hline \multicolumn{4}{|l|}{ Residence, $n(\%)$} \\
\hline Urban & $59(73.7)$ & $105(61.0)$ & \multirow{2}{*}{$<0.001$} \\
\hline Rural & $21(26.3)$ & $67(39.0)$ & \\
\hline Onset in daytime (6:00-18:00) & $58(72.5)$ & $77(44.8)$ & $<0.001$ \\
\hline \multicolumn{4}{|l|}{ Etiology of ischemic stroke, $n(\%)$} \\
\hline Large artery atherosclerosis & $33(41.3)$ & $64(37.2)$ & \multirow{5}{*}{0.011} \\
\hline Small artery occlusion & $26(32.5)$ & $70(40.7)$ & \\
\hline Cardioembolism & $20(25.0)$ & $29(16.9)$ & \\
\hline Other demonstrated cause & 0 & $4(2.3)$ & \\
\hline Undetermined cause & $1(1.2)$ & $5(2.9)$ & \\
\hline NIHSS at admission, median (IQR) & $8(3-14)$ & $3(2-7)$ & 0.031 \\
\hline Stroke history, $n(\%)$ & $19(24.1)$ & $36(21.1)$ & 0.523 \\
\hline Hypertension, $n(\%)$ & $53(66.3)$ & $107(62.2)$ & 0.278 \\
\hline Diabetes, $n(\%)$ & $36(45.0)$ & $70(40.7)$ & 0.298 \\
\hline Hyperlipidemia, $n(\%)$ & $25(31.3)$ & $56(32.6)$ & 0.776 \\
\hline Atrial fibrillation, $n(\%)$ & $12(15.0)$ & $23(13.3)$ & 0.165 \\
\hline Coronary heart disease, $n(\%)$ & $12(15.0)$ & $27(15.7)$ & 0.679 \\
\hline Current smoker, $n(\%)$ & $36(45.0)$ & $72(42.0)$ & 0.108 \\
\hline Regular drinker, $n(\%)$ & $32(40.0)$ & $63(36.7)$ & 0.182 \\
\hline Self-management after onset, $n(\%)$ & $8(10.0)$ & $118(68.6)$ & $<0.001$ \\
\hline Transported by ambulance, $n(\%)$ & $38(43.7)$ & $57(33.1)$ & 0.018 \\
\hline Post-COVID-19 period, $n(\%)$ & $22(27.5)$ & $69(38.9)$ & 0.015 \\
\hline
\end{tabular}

IQR, interquartile range; NIHSS, National Institutes of Health Stroke Scale; ONT, onset-to needle time.

ic $(41.1 \%$ vs. $63.6 \% ; p<0.001)$. The proportion of patients from onset to treatment within $4.5 \mathrm{~h}$ was significantly decreased after the COVID-19 pandemic ( 34.8 vs. $29.0 \%$, $p=0.032$ ). The proportion of patients with intravenous thrombolysis was decreased significantly after the pandemic ( 20.1 vs. $15.4 \%, p=0.030$ ), while the proportion of patients with mechanical thrombectomy (15.7 vs. $12.1 \%$, $p=0.115$ ), in-hospital mortality (4.9 vs. $6.1 \%, p=0.120$ ), and discharge mRS score (0-2) remained unchanged (60.1 vs. $54.5 \%, p=0.180$, Table 1 ).

When compared with patients who arrived to the hospital within $4.5 \mathrm{~h}(\mathrm{ONT} \leq 4.5 \mathrm{~h})$, those who arrived to the hospital latter (ONT $>4.5 \mathrm{~h}$ ) had a lower education level (elementary education: 18.4 vs. $37.2 \%, p=0.018$ ), more likely lived in rural areas $(26.3$ vs. $39.0 \%, p<0.001)$ and living alone (26.3 vs. $33.7 \%, p=0.044)$, more likely had small artery occlusion ( 32.5 vs. $40.7 \%, p=0.011$ ), had lower NIHSS scores ( 8 vs. $3, p=0.031$ ), less likely transferred by ambulance ( 43.7 vs. $16.1 \%, p<0.001$ ), more likely had self-management after stroke onset (9.2 vs. $87.8 \%, p<0.001)$, less likely ( 72.5 vs. $44.8 \%, p<0.001)$ had daytime onset (6:00-18:00), and more likely had stroke after COVID-19 pandemic (27.6 vs. $45.6 \%, p=0.031$, Table 2).

Table 3 presents the potential influencing factors for delayed treatment $(\mathrm{ONT}>4.5 \mathrm{~h}$ ) by multivariate logistic regression analysis. Compared with patients before COVID-19 pandemic, patients after COVID-19 pandemic had an odds ratio (OR) of 1.52 (95\% confidence interval [CI], 1.02-2.94) for treatment delay. Compared with patients with advanced education, those with elementary education had an OR of 1.41 (95\% CI, 1.08-2.31) for 
Table 3. Multivariate logistic regression analysis of influencing factors for delayed treatment $(\mathrm{ONT}>4.5 \mathrm{~h})$

\begin{tabular}{lrrr}
\hline Variables & OR & 95\% CI & $p$ value \\
\hline Elementary versus higher education & 1.41 & $1.08-2.31$ & 0.045 \\
Rural versus urban residency & 1.20 & $1.01-1.42$ & 0.030 \\
High NIHSS score & 0.64 & $0.45-0.89$ & $<0.001$ \\
Small artery occlusion & 2.44 & $1.28-3.75$ & $<0.001$ \\
Self-management after onset & 2.03 & $1.40-3.76$ & $<0.001$ \\
Transported by ambulance & 0.76 & $0.68-0.86$ & 0.038 \\
Onset after COVID-19 pandemic & 1.52 & $1.02-2.94$ & 0.010 \\
\hline
\end{tabular}

ONT, onset-to-needle time; OR, odds ratio; CI, confidence interval; NIHSS, National Institutes of Health Stroke Scale.

treatment delay. Compared with patients living in urban areas, those living in rural areas had an OR of 1.20 (95\% CI, 1.01-1.42) for treatment delay. Patients who had a small artery occlusion had an OR of 2.44 (95\% CI, 1.283.75 , vs. other types of ischemic stroke) for treatment delay. Patients with baseline a high NIHSS score had an OR of 0.64 (95\% CI, 0.45-0.89) for treatment delay. Patients who first chose for self-management after stroke onset had an OR of 2.03 (95\% CI, 1.40-3.76) for treatment delay. Patients transported by ambulance had an OR of 0.76 (95\% CI, 0.68-0.86) for treatment delay.

\section{Discussion}

The current study highlights the impact of the COVID-19 pandemic on treatment delay in patients with acute stroke. ODT and DNT were significantly prolonged, and the proportion of patients who arrived within the time window for intravenous thrombolysis was significantly decreased after the COVID-19 pandemic.

During COVID-19, patients may be reluctant to seek medical help for fear of being infected. Patients with mild symptoms may stay at home and manage stroke by themselves or their relatives. This speculation was partly confirmed by the higher NIHSS score in post-COVID-19. A similar pattern of delay in seeking medical care due to fear of being infected within the hospitals was observed in the Ebola epidemic in West Africa [6].

During the COVID-19 pandemic, the ONT was significantly prolonged than before. Traffic control during the pandemic may delay the patient transportation. Social distance may influence the management of stroke patients. Procedures for COVID-19 prevention, such as in- formation inquiring concerning travel and contact history, temperature measuring, chest X-ray or computed tomography scanning, coronavirus nucleic acid or antibody testing, blood cell counting, and multidisciplinary consultation may all delay the management of stroke. On the other hand, medical staff may need longer time to wear protective devices before they could manage stroke patients during the COVID-19 pandemic.

This study associated a higher NIHSS score with shorter prehospital delay. This is consistent with some previous studies [7, 8] but not with others [9]. Patients with severe symptoms may be more obvious to be identified, but severe symptoms may render patients from seeking for help when alone. Stroke patients with small vessel disease were referred later than stroke patients with other etiologies. It may be partly attributed to the mild neurologic deficit in patients with AIS caused by small vessel disease. Another potential explanation lies in that vascular cognitive impairment, which may prevent patients from going to hospital, which is more common in patients with small vessel disease.

Transferred with ambulance was associated with shorter prehospital delay [10-13]. Early awareness of stroke onset and rapid response is crucial to shorten the treatment delay $[14,15]$. Previous studies [16] indicated that the major factors for prehospital delay included unawareness of stroke symptoms, lack of understanding on importance of early response, and lack of knowledge on early management. Previous studies demonstrated that only $15.6 \%$ of respondents knew stroke warning signs [17]. A large proportion of respondents think that stroke symptoms may not warrant emergent management [18].

The American Heart Association and American Stroke Association Temporary Emergency Guidance to US Stroke Centers During the COVID-19 Pandemic recommended telemedicine to help neurologists collect medical history without contacting patients, reducing contact risk, saving time, and isolating equipment. At the same time, telephone/video consultation can help patients choosing the most appropriate hospital at the first time, avoiding additional exposure risk of COVID-19 and shortening prehospital delay [19].

For posthospital management of stroke patients, guidelines recommended that the green channel should be equipped with personal protective equipment (PPE) and continue to promote intravenous thrombolysis and endovascular therapy in accordance with the regular procedures [19]. Other posthospital efforts include optimizing the process of wearing PPE, conducting simulation training, shortening the wearing time of PPE, and opti- 
mizing communication and co-operation among departments to reduce the examination time before thrombolysis and thrombectomy.

Several limitations should be addressed when interpreting the results of the current study. First, patients were enrolled outside the epicenter of the COVID-19 pandemic in China, which may have underestimated the impacts of the pandemic on stroke management. Second, with the accumulation of coping experiences, the impacts of the COVID-19 pandemic on stroke management may be relieved. Finally, only 2 hospitals were included in this study. There might be selection bias, although patients were enrolled consecutively. Multicenter collaborations and population-based studies are warranted to draw a complete picture of the subject.

\section{Conclusion}

In conclusion, COVID-19 pandemic has a remarkable influence on the management of AIS. In Jiangsu province where this study was carried out, social distance, quarantine, pathogen testing, suspension of classes, and temporarily shutting-down of factories were implemented during COVID-19 pandemic. These strategies were also implemented in other provinces of China and many other countries. Both pre- and posthospital delays were prolonged significantly, and the proportion of patients arrived within the 4.5-h time window for intravenous thrombolysis treatment was decreased. Given that antiCOVID-19 measures are becoming medical routines, efforts are warranted to shorten the delay so that the stroke outcome could be improved in this complex time.

\section{Acknowledgements}

The authors acknowledge all consulting neurologists in Jinling Clinical College of Nanjing Medical University and the affiliated Yixing Hospital of Jiangsu University.

\section{Statement of Ethics}

This retrospective study was approved by the local Ethics Committee of the University of Jiangsu (20-2021-001) and performed in accordance with the guidelines of the World Medical Association Declaration of Helsinki. All the participants and their relatives provided written informed consent.

\section{Conflict of Interest Statement}

The authors have no conflicts of interest to declare. A preprint version of this article is available at medRxiv [20].

\section{Funding Sources}

The work was supported by (National Natural Science Foundation of China) grant numbers (81870947).

\section{Author Contributions}

S.G. and Z.D.: study design, interpretation of results, and manuscript drafting. S.G., Y.B., and H.S.: study design and interpretation of results. S.G., Z.D., Y.B., H.S., and X.Z.: data collection. G.X. and S.G.: study design, statistical analysis, and critical revision of the manuscript. S.G.: interpretation of results and critical revision of the manuscript. G.X. and X.L. have full access to all of the data in the study and take responsibility for the integrity of the data and the accuracy of the data analysis.

\section{References}

1 Hacke W, Kaste M, Bluhmki E, Brozman M, Dávalos A, Guidetti D, et al. Thrombolysis with alteplase 3 to 4.5 hours after acute ischemic stroke. N Engl J Med. 2008;359(13): 1317-29.

2 Nogueira RG, Jadhav AP, Haussen DC, Bonafe A, Budzik RF, Bhuva P, et al. Thrombectomy 6 to 24 hours after stroke with a mismatch between deficit and infarct. N Engl J Med. 2018;378(1):11-21.

3 William J, Alejandro A, Teri A, Opeolu M, Nicholas C, Kyra B. 2018 guidelines for the early management of patients with acute ischemic stroke. Stroke. 2018;49:e6-e99.

4 Cucinotta D, Vanelli M. WHO declares COVID-19 a pandemic. Acta Biomed. 2020; 91(1):157-60.
5 Yang B, Wang T, Chen J, Chen Y, Wang Y, Gao P, et al. Impact of the COVID-19 pandemic on the process and outcome of thrombectomy for acute ischemic stroke. J Neurointerv Surg. 2020.

6 McQuilkin PA, Udhayashankar K, Niescierenko M, Maranda L. Health-care access during the ebola virus epidemic in Liberia. Am J Trop Med Hyg. 2017;97(3):931-6.

7 Jin H, Zhu S, Wei JW, Wang J, Liu M, Wu Y, et al. Factors associated with prehospital delays in the presentation of acute stroke in urban China. Stroke. 2012;43(2):362-70.

8 Fang J, Yan W, Jiang GX, Li W, Cheng Q. Time interval between stroke onset and hospital arrival in acute ischemic stroke patients in Shanghai, China. Clin Neurol Neurosurg. 2011;113(2):85-8.
9 Huang Q, Ma QF, Jia JP, Feng J, Cheng WY, Chang $\mathrm{H}$, et al. Referral leads to prehospital delay of intravenous thrombolysis for acute ischemic stroke in Beijing. Int J Stroke. 2015; 10(7):E80-1.

10 Minnerup J, Wersching H, Unrath M, Berger $K$. Effects of emergency medical service transport on acute stroke care. Eur J Neurol. 2014; 21(10):1344-7.

11 Jiang B, Ru X, Sun H, Liu H, Sun D, Liu Y, et al. Pre-hospital delay and its associated factors in first-ever stroke registered in communities from three cities in China. Sci Rep. 2016;6(1):29795. 
12 Yang H, Zhang J, Xie J, Yang C, Dong X, Gong $\mathrm{Y}$, et al. Factors influencing pre-hospital delay among acute ischemic stroke patients in the midlands of China. Int J Cardiol. 2014;172(2): 533-4.

13 Price CI, Rae V, Duckett J, Wood R, Gray J, McMeekin P, et al. An observational study of patient characteristics associated with the mode of admission to acute stroke services in North East, England. PloS One. 2013;8(10): e76997.

14 Bouckaert M, Lemmens R, Thijs V. Reducing prehospital delay in acute stroke. Nat Rev Neurol. 2009;5(9):477-83.
15 Mattew J. Reducing the delay between stroke onset and hospital arrival: is it an achievable goal? J Am Heart Assoc. 2012;1:e002477.

16 Sun H, Chen S, Jiang B, Zhao X, Wu S, Liu Y, et al. Public knowledge of stroke in Chinese urban residents: a community questionnaire study. Neurol Res. 2011;33(5):536-40.

17 Yang J, Zheng M, Cheng S, Ou S, Zhang J, Wang N, et al. Knowledge of stroke symptoms and treatment among community residents in Western Urban China. J Stroke Cerebrovasc Dis. 2014;23(5):1216-24.
18 Le Bonniec A, Haesebaert J, Derex L, Porthault S, Préau M, Schott AM. Why patients delay their first contact with health services after stroke? A qualitative focus group-based study. PloS One. 2016;11(6):e0156933.

19 Patrick L. Temporary emergency guidance to US stroke centers during the coronavirus disease 2019 (COVID-19) pandemic: on behalf of the American heart association/American stroke association stroke council leadership. Stroke. 2020;51(6):1910-2.

$20 \mathrm{Gu}$ S, Dai Z, Shen H, Bai Y, Zhang X, Liu X, et al. Delayed stroke treatment during $\mathrm{CO}$ VID-19 pandemic in China. Medrxiv. 2020. 